\title{
DYNAMIC STABILITY OF COMPACT STARS
}

\author{
G.S.Bisnovatyi-Kogan \\ Space Research Institute RAN, Moscow, Russia \\ and \\ Joint Institute of Nuclear Researches, Dubna, Russia * \\ gkogan@mx.iki.rssi.ru
}

\begin{abstract}
After some historical remarks we discuss different criteria of dynamical stability of stars, and properties of the critical states where dynamical stability is lost, leading to collapse with formation of the neutron star or a black hole. At the end some observational and theoretical problems related to quark stars are discussed.
\end{abstract}

Keywords: stellar stability, white dwarfs, neutron stars, quark stars

\section{Introduction}

It was first noted by S Chandrasekhar in 1931 that the mass of the compact star at very large densities, when electrons become relativistically degenerate cannot exceed a value, which is now called as Chandrasekhar limit for white dwarfs (Chandrasekhar, 1931). After discovery of the neutron in 1932 the idea of the neutron star existence was developed. The matter in these stars is so dense, that neutrons become relativistically degenerate, again leading to the maximum mass of the neutron star. There are two important differences between white dwarfs and neutron stars, influencing the stability and mass limit. Neutron stars are about 6 orders of magnitude denser, than white dwarfs, making necessary to use general relativity (GR) in description of gravitation, instead of newtonian gravity. In GR the gravitation force is growing faster than $1 / r^{2}$, what reduce a stability and decrease the mass limit. On the other hand neutrons interaction become important at densities exceeding the nuclear one. The repulsion forces between nucleons at large densities

*Partial funding provided by RFBR grant 02-02-16900. 
lead to additional stabilization against collapse, increasing the limiting mass of the neutron star. The influence of the second factor occurs to be more important, so at present the limiting mass of the neutron star is taken about two times larger than that of the white dwarf.

Discovery of the quark structure of the matter led to suggestion of possible existence of quark stars, even more compact than neutron ones. In presence of indefiniteness concerning quark structure of the matter it is not possible now to make definite statements about possibility of existence or nonexistence of stable quark stars, observational and theoretical investigations on this topic are still in progress.

In this review I first make a historical excursus into the problem, mentioning the results of the key works. Several criteria of stability are discussed, with the main attention to the static criteria, and energetic method, which permit to obtain the conclusion about the stability (sometimes approximately) in a most simple way. Critical states of compact stars at the boundary of the dynamic stability are considered, at which the star is becoming instable in the process of energy losses, and collapse begins leading to formation of the neutron star or a black hole. Physical processes leading to a loss of stability are discussed. At the end some observations and theoretical problems connected with quark stars are considered.

\section{Early development of the theory of compact stars: 1931-1965}

From the theory of polytropic gravitating gas spheres with the equation of state $P=K \rho^{\gamma}$ it was known (Emden, 1907), that at $\gamma=4 / 3$ the equilibrium mass has a unique value

$$
M=4\left(\frac{K \pi}{G}\right)^{3 / 2} M_{3},
$$

where $M_{3}=2.01824$ is a non-dimensional mass, corresponding to the polytropic index $n=3, \gamma=1+\frac{1}{n}$. Such a star is in a neutral equilibrium at any density. Chandrasekhar (1931) first noticed, that at large densities the electron degeneracy is becoming ultrarelativistic with the equation of state

$$
P=\frac{\pi}{4}\left(\frac{3}{\pi}\right)^{1 / 3} \hbar c n_{\mathrm{e}}^{4 / 3}, \quad n_{\mathrm{e}}=\frac{\rho}{\mu m_{p}} .
$$

For some unknown reason Chandrasekhar (1931) took " $\mu$ equals the molecular weight, 2.5, for a fully ionized material", corresponding to $K=3.619 \cdot 10^{14}$ and had obtained the limiting mass from (1) equal 
to $1.822 \cdot 10^{33} \mathrm{~g}=0.91 M_{\odot}$. Landau (1932) noticed, that for most stable nuclei: $\mathrm{He}^{4}, \mathrm{C}^{12}, \mathrm{O}^{16}$ etc. the value of $\mu$, which is the number of nucleons on one electron, is equal to 2 , and he obtained the realistic value of the limiting mass $M_{\text {lim }}=1.4 M_{\odot}$.

Soon after the neutron discovery (Chadwick, 24 Feb. 1932, letter to N.Bohr), "Landau improvised the concept of neutron star" in discussion with Bohr (Rosenfeld, 1974). The modern conception of the neutron star origin was due to Baade and Zwicky (1934), which appeared as a result of their studies of supernovae explosions. Their conclusion: "With all reserve we advance the view that supernovae represent the transitions from ordinary stars into neutron stars, which in their final stages consist of extremely closely packed neutrons", is an example of classic astrophysical predictions, finally proved only 35 years later after discovery of the pulsar in the center of the Crab nebula (Lovelace et al., 1968; Comella et al., 1969). Investigation of properties of matter at nuclear and larger densities expected in neutron stars had been started in papers of Hund (1936), Landau (1938) and Gamow (1938).

The first neutron star model in GR was constructed by Oppenheimer and Volkoff (1939). They have derived equation of spherically symmetric stellar equilibrium in GR using metric in the form

$$
d s^{2}=-e^{\nu} d t^{2}+e^{\lambda} d r^{2}+r^{2}\left(d \theta^{2}+\sin ^{2} \theta d \varphi^{2}\right)
$$

with equilibrium equations

$$
\begin{gathered}
\frac{d P}{d r}=-\frac{G\left(\rho+P / c^{2}\right)\left(m+4 \pi r^{3} P / c^{2}\right)}{r^{2}\left(1-2 G m / r c^{2}\right)}, \\
\frac{d m}{d r}=4 \pi \rho r^{2}, \quad \rho=\rho_{0}\left(1+\frac{E}{c^{2}}\right), \quad \rho(R)=0, \quad M=m(R) .
\end{gathered}
$$

An ideal degenerate neutron gas at zero temperature was considered with energy $E_{n}$, pressure $P_{n}$, and rest mass density $\rho_{0}$ as

$$
\begin{gathered}
E_{n}=\frac{m_{n}^{4} c^{5}}{24 \pi^{2} \hbar^{3} \rho} g(y)=\frac{6.860 \cdot 10^{35}}{\rho} g(y), \\
P_{n}=\frac{m_{n}^{4} c^{5}}{24 \pi^{2} \hbar^{3} \rho_{0}} f(y)=\frac{6.860 \cdot 10^{35}}{\rho_{0}} f(y), \\
\rho_{0}=\frac{m_{n}^{4} c^{3}}{3 \pi^{2} \hbar^{3}} y^{3}=6.105 \cdot 10^{15} y^{3}
\end{gathered}
$$

where 


$$
\begin{gathered}
f(y)=y\left(2 y^{2}-3\right) \sqrt{y^{2}+1}+3 \sinh ^{-1} y \\
g(y)=3 y\left(2 y^{2}+1\right) \sqrt{y^{2}+1}-3 \sinh ^{-1} y, \quad y=\frac{p_{n, F e}}{m_{n} c} .
\end{gathered}
$$

They had obtained the following parameters of the neutron stars of different masses:

Table 1. Total mass of the neutron star model versus its radius, calculated by Oppenheimer and Volkoff (1939).

\begin{tabular}{||c||c||}
\hline Total mass in Solar units & Radius in $\mathrm{km}$ \\
\hline \hline 0.30 & 21.1 \\
0.60 & 13.3 \\
0.71 & 9.5 \\
0.64 & 6.8 \\
0.34 & 3.1 \\
\hline
\end{tabular}

Between white dwarfs and neutron star there is a neutronization and transition from normal matter with electrons and nuclei to superdense matter consisting of neutrons and other strongly interacting particles: mesons and adrons. The continuous curve $M\left(\rho_{c}\right)$ in the whole region from white dwarfs to neutron stars was first constructed by Wheeler (1958), where only neutrons have been considered in the superdense phase, like in Oppenheimer and Volkoff (1939). The results of Wheeler (1958) are given in Fig.1. The first neutron star model with a realistic equation of state, including mesons, hyperons and nuclear interaction, was constructed by Cameron (1959). The following equilibrium reactions between strongly interacting particles had been taken into account

$$
\begin{gathered}
n+n \rightarrow n+n+2 \pi^{0} \rightarrow \Lambda^{0}+\Lambda^{0}-2(177) \mathrm{MeV}, \\
p+p \rightarrow p+p+2 \pi^{0} \rightarrow \Sigma^{+}+\Sigma^{+}-2(251) \mathrm{MeV}, \\
\Lambda^{0}+\Lambda^{0} \rightarrow \Lambda^{0}+\Lambda^{0}+\pi^{0} \rightarrow \Sigma^{0}+\Sigma^{0}-2(77) \mathrm{MeV} \\
n+n \rightarrow n+p+\pi^{-} \rightarrow p+\Sigma^{-}-256 \mathrm{MeV} \\
n+\Lambda^{0} \rightarrow p+\pi^{-}+\Lambda^{0} \rightarrow p+\Xi^{-}-204 \mathrm{MeV}, \\
\Lambda^{0}+\Lambda^{0} \rightarrow \Lambda^{0}+\Lambda^{0}+2 \pi^{0} \rightarrow \Xi^{0}+\Xi^{0}-2(195) \mathrm{MeV} .
\end{gathered}
$$

The nuclear interaction was considered according to Skryme (1959), see Fig.2. This equation of state is not perfect, because at large densities it 
violates the casuality principle, according to which the sound speed cannot exceed the light speed (see Zeldovich, 1961). Neutron star models obtained by Cameron (1959) are represented in Fig.3. The most important result of this work was the indication that at realistic equation of state the limiting mass of the neutron star may exceed the Chandrasekhar mass limit for white dwarfs. Therefore after a loss of stability a stellar core with mass exceeding the Chandrasekhar mass limit may stop its contraction forming a stable neutron star with enormous energy output, according to Baade and Zwicky (1934). In the model of Oppenheimer and Volkoff (1939) the collapse would not stop, because of the low mass limit of the neutron star.

Cameron (1959) had obtained that models at high densities had monotonously decreasing mass asymptotically tending to a constant value. That was probably the result of a crudeness of calculations, because the behavior of the curve $M\left(\rho_{c}\right)$ at large densities has oscillating, and not monotonous dependence. Such oscillations had been present in calculations of Ambartsumyan and Saakyan (1961), who also improved physical description of superdense matter (Ambartsumyan and Saakyan, 1960). The nature of high density oscillations of mass in GR equilibrium was explained by Dmitriyev and Kholin (1963) (see also Misner and Zapolsky, 1964). They had shown that all models beyond the first neutron star mass maximum on the curve $M\left(\rho_{c}\right)$ are unstable, and after each new extremum the model acquires one new unstable mode. Later this problem was analyzed at length in the book of Harrison et al. (1965). Detailed calculations of neutron star models with a realistic equation of state had been done by Saakyan and Vartanyan (1964).

\section{Criteria of hydrodynamic stability}

The exact approach to the problem of dynamic (linear) stability is based on the solution of the equations for small perturbations, and finding eigenvalues and eigenfunctions of these equations. In the conservative system the variation principle may be derived, which determines the exact value of eigenfrequency if the exact eigenfunction is known. In practice even use of an approximate linear eigenfunction often define the eigenfrequency with a good precision. The variation principle for a spherically symmetric star in GR was first derived by Chandrasekhar (1964) from the equations of small perturbations, and later by Harrison et al. (1965) by variation of the potential energy of the star. The exact method for determination of the dynamic stability of the star to the

adiabatic perturbations based on the sequence of static solutions was formulated by Zeldovich (1963). He had shown, that in the extremum of 


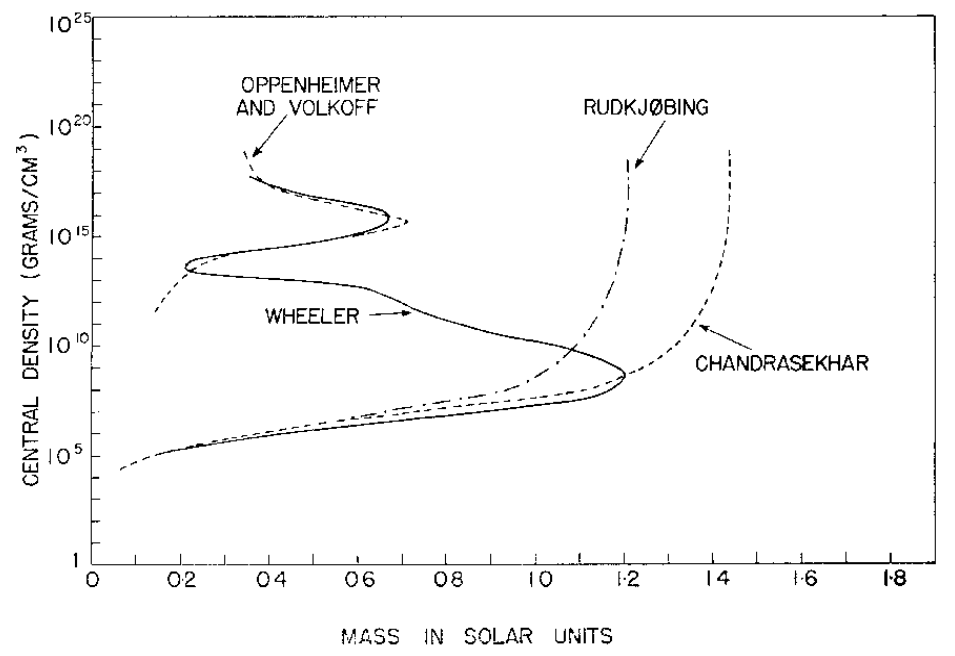

Figure 1. The relation between central density and the mass of various degenerate star models. Chandrasekhar's curve is for white dwarfs with a mean molecular weight 2 of atomic mass units. Rudkjóbing's curve is the same except for inclusion of the relativistic spin-orbit effects Rudkjóbing (1952). The curve labeled "Oppenheimer and Volkoff" is for a set of neutron star models. The solid line marked "Wheeler" is a set of models computed with a generalized equation of state, from Cameron (1959)

the function $M\left(\rho_{c}\right)$, or $M(R)$ along the sequence with a constant entropy $S$ adds or subtract one unstable mode. On the curve of cold stars on Fig. 1 the stability is lost in the first maximum due to neutronization, or GR effects, but it is restored in the minimum, where stable neutron star appears. The static criterium of stability was generalized for the case of arbitrary rotating stars with arbitrary distribution of entropy over the star in the paper of Bisnovatyi-Kogan and Blinnikov (1974). It was noted, that in presence of three quantities conserved during adiabatic oscillations, the critical points are the extrema of three functions $\left.M\left(\rho_{c}\right)\right|_{s, j}$, $\left.s\left(\rho_{c}\right)\right|_{M, j}, J\left(\rho_{c}\right) \mid M, s$. Here along the sequences not only total values of stellar angular momentum $J$ and entropy $S$ should be conserved, but also their distributions over the lagrangian coordinate $N$, which is the number of baryons inside the cylindrical radius, $j(N)$ and $s(N)$. Note that in the newtonian case lagrangian coordinate is coincides with lagrangian mass $m=\rho N=\rho_{0} N$, but differs from it in GR case. For polytropic equation of state and angular momentum distribution similar to rigid rotation 


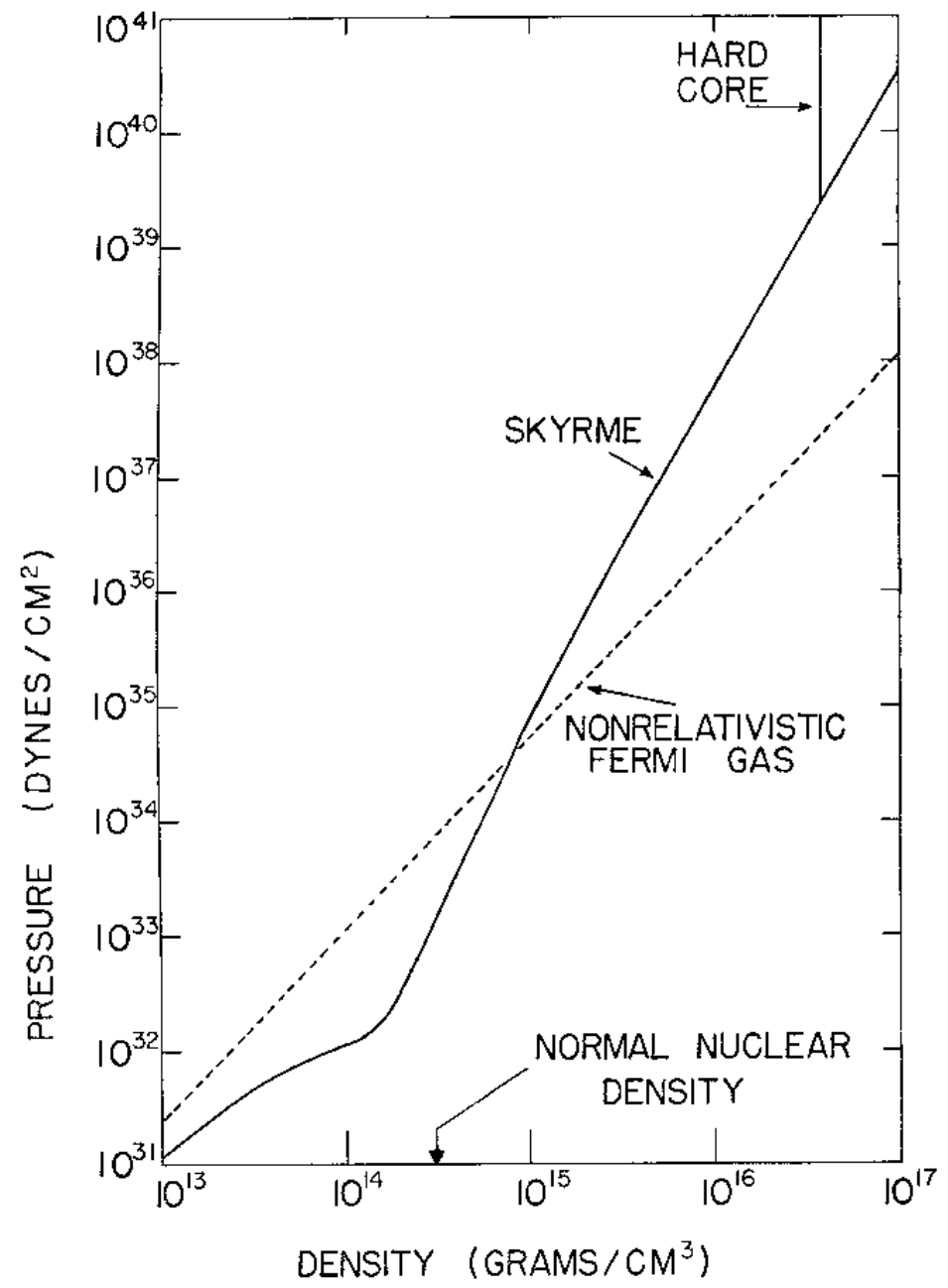

Figure 2. Skryme's equation of state. The hard core modification is shown at the upper right, and the dashed line is the equation of state for a non-relativistic Fermi gas of neutrons, from Cameron (1959).

$$
P=K \rho^{1+1 / n}, \quad K=\mathrm{const} \cdot e^{s}, \quad j(m)=\frac{5}{2} \frac{J}{M}\left[1-(1-m)^{2 / 3}\right]
$$

examples of application of the static criteria are represented in Fig. 4 from Bisnovatyi-Kogan and Blinnikov (1974).

Let us stress, that along the curve appropriate for a static criterium of stability the rotation does not remain rigid, but only angular momentum of each lagrangian loop should be conserved. It means that if you have a curve of rigidly rotating stars with a fixed total angular momentum you 


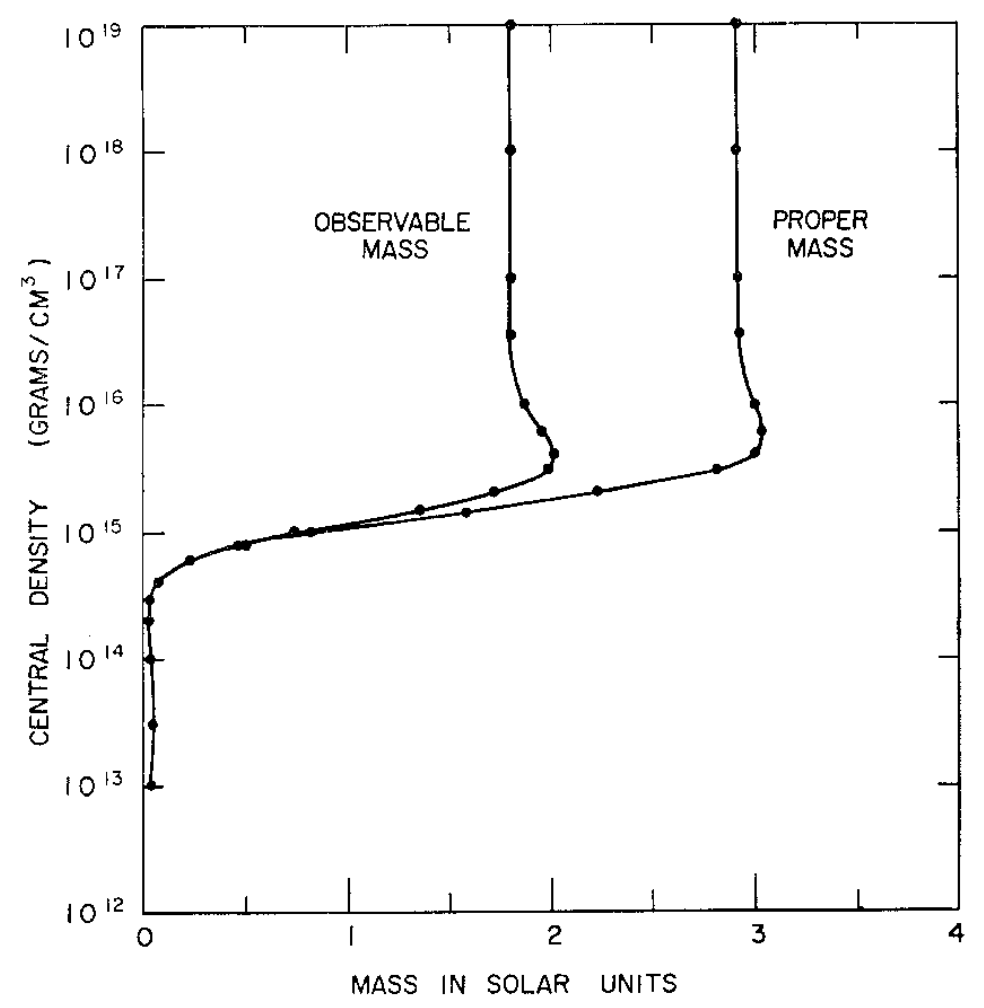

Figure 3. The observable and proper masses of neutron star models with non-ideal matter, from Cameron (1959).

should construct an adjusted curve from each point of this curve with a constant angular momentum of a lagrangian loop, which maximum indicated a loss of stability. The rigidly rotating model is on the border of stability, when coincides with the maximum of the adjusted curve. For a cold white dwarf with the equation of state

$$
\begin{gathered}
\rho=A x^{3}\left(2+a_{1} x+a_{2} x^{2}+a_{3} x^{3}\right), \\
P=B\left[x\left(2 x^{2}-3\right)\left(x^{2}+1\right)^{1 / 2}+3 \ln \left(x+\sqrt{1+x^{2}}\right)\right], \\
A=9.82 \times 10^{5} \mathrm{~g} \mathrm{~cm}^{-3}, \quad B=6.01 \times 10^{22} \mathrm{erg} \mathrm{cm}^{-3}, \\
a_{1}=1.255 \times 10^{-2}, \quad a_{2}=1.755 \times 10^{-5}, \quad a_{3}=1.376 \times 10^{-6},
\end{gathered}
$$

the adiabatic index is close to $n=3$ at the critical point, and the eigenfunction is close the the linear one. At homologous transformation 

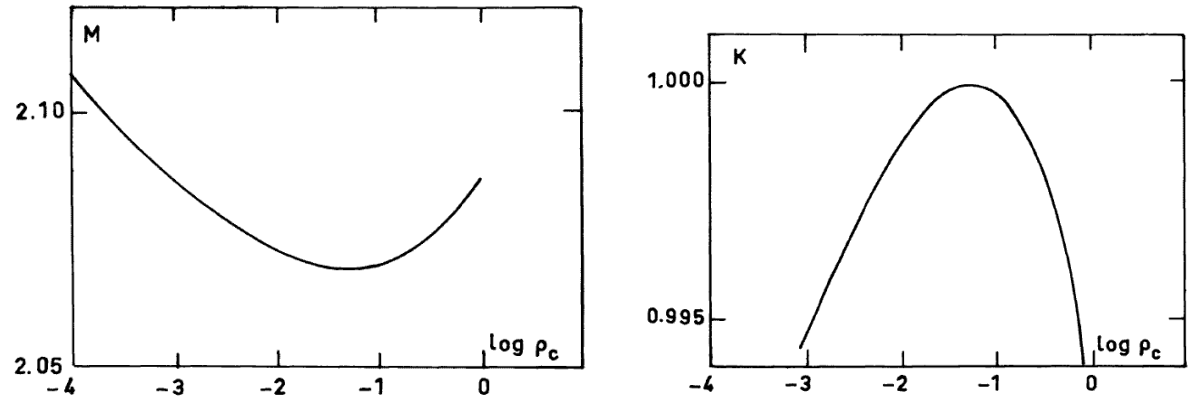

Figure 4. The dependence of the mass $M$ on the central density for fixed entropy and angular momentum, for isentropic models having a polytropic index $n=3.03$. The angular momentum distribution is given in (8) (left). The dependence of $K$ on central density for fixed angular momentum distribution, corresponding to the minimum of mass in the left figure, for isentropic models with polytropic index $n=3.03$ (right). From Bisnovatyi-Kogan and Blinnikov (1974).

the rigid rotation is preserved, so the adjusted curve for white dwarfs differs only slightly from the curve of rigidly rotating models, see Fig.5 (left). For a more complicated equation of state with highly variable adiabatic index, like in the equation of state

$$
\begin{gathered}
\rho(H)=\frac{1}{2} \rho_{1}\left[\left(\frac{H}{H_{1}}\right)^{0.3}+\left(\frac{H}{H_{1}}\right)^{10}\right], \\
P(H)=\frac{1}{2} \rho_{1} H_{1}\left[\frac{\left(H / H_{1}\right)^{1.3}}{1.3}+\frac{\left(H / H_{1}\right)^{11}}{11}\right] .
\end{gathered}
$$

the value $\gamma_{1}=4 \frac{1}{3}$ at $\rho=\rho_{1}$ and falls off steeply when $\rho>\rho_{1}$. The results of calculations for a model with solid-body rotation and three associated series of models are given in Fig.5 (right). The loss of stability occurs at the point of intersection of the associated curve $D$ with the solid-body one, this point coinciding with the maximum on the curve $D$. It can be seen from Fig.5 (right) that the point of stability loss differs from the maximum on the solid-body curve by almost $5 \%$ with respect to $\rho_{c}$. An extension of static criteria to general relativity and toroidal magnetic fields is made in the paper of Bisnovatyi-Kogan and Blinnikov (1974). Static criteria remains valid also in the presence of phase transitions. Variational principle and other methods of investigation of stability in presence of phase transitions are considered by Bisnovatyi-Kogan, Blinnikov and Shnol (1975). The review of stellar stability methods is given in the book of Bisnovatyi-Kogan (2002). 

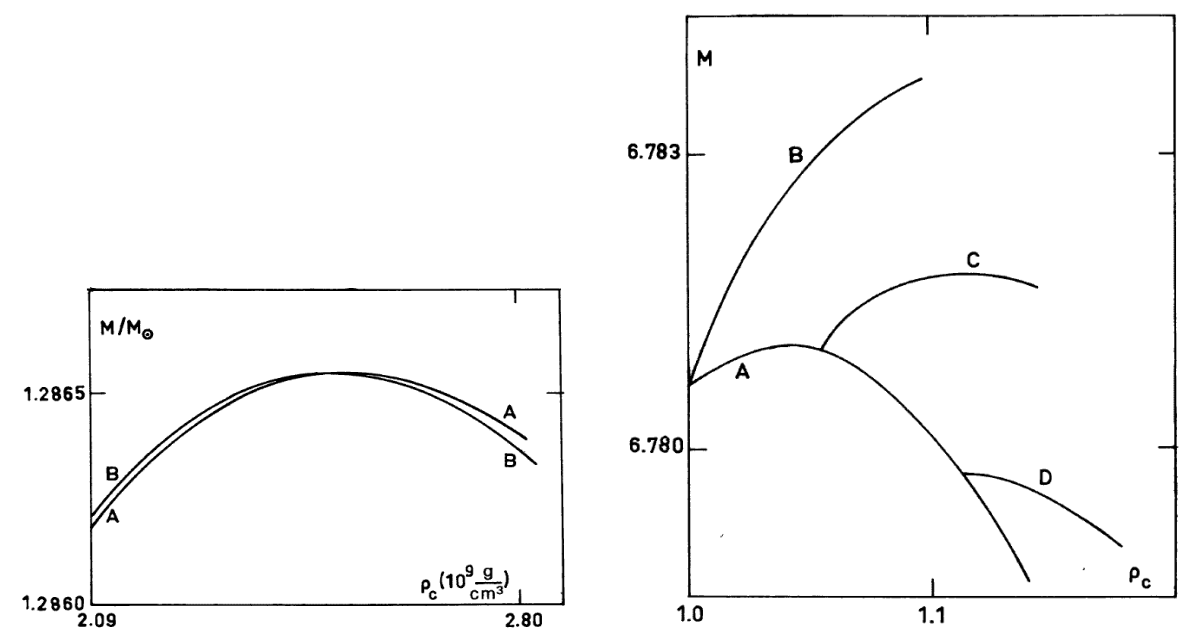

Figure 5. The dependence of the mass $M$ on the central density for rigidly (Curve A) and differentially (Curve B) rotating white dwarfs with the equation of state from (9). The angular momentum distribution of the Curve B is that of the extremal model of Curve A (left). The dependence of the mass $M$ on the central density for rigidly (Curve A) and differentially (Curves B,C and D) rotating models with the equation of state from (10). The angular momentum distribution of the Curves $\mathrm{B}, \mathrm{C}$ and $\mathrm{D}$ are those of the rigidly rotating models of the Curve $\mathrm{A}$ at the points of its intersection with the Curves B,C and D. The curve C is intersecting the Curve A in its maximum. The maximum of the Curve D coincides with the point of intersection, which is a critical point for rigidly rotating models.(right). From Bisnovatyi-Kogan and Blinnikov (1974).

\section{Energetic method}

In the case of a complicated equation of state and entropy distribution along the star, the static criteria is hard to apply. In this case it is more convenient to use an approximate energetic method, which follows from the exact variation principle where linear trial function is used for estimation of the eigenfrequency.

Energetic method was first applied for investigation of stability of supermassive isentropic stars by Zeldovich and Novikov (1965) where first GR corrections to the energy of such star have been found. This method was generalized for arbitrary stars by Bisnovatyi-Kogan (1966). Equations have been derived, giving approximate description of equilibrium and determining the critical point where stability is lost. The first equation represents equilibrium condition, following from first variation of the total potential energy of that star (gravitational plus internal for nonrotating stars) equal to zero. The model with adiabatic index $n=3$, 
$\gamma=4 / 3$ is in a neutral stability with a linear eigenfunction. In application of the energetic method the density distribution of the model is prescribed by the polytropic $n=3$ distribution, $\rho=\rho_{c} \varphi(m / M)$, where $m$ is lagrangian mass coordinate, and $\varphi(m / M)$ is connected with the Emden function corresponding to $n=3$, with a linear trial function $\delta r=\alpha r, \alpha=$ const. The equation of state is arbitrary with the prescribed entropy distribution over the lagrangian coordinate, and first GR correction to the energy, including all terms $\sim R / R_{g}, R$ is stellar radius, $R_{g}=2 G M / c^{2}$ is the gravitational radius, are taken into account. The equilibrium equation in the approximate energetic method is:

$$
3 \rho_{c}^{4 / 3} \int_{0}^{M} P \frac{d m}{\varphi\left(\frac{m}{M}\right)}-0.639 G M^{5 / 3}-1.84 \frac{G^{2} M^{7 / 3}}{c^{2}} \rho_{c}^{1 / 3}=0 .
$$

Under the above mentioned conditions the second variation of the energy is also reduced to the integral relation. Zero value of this relation approximately corresponds to the critical state of the loss of stability. We have

$$
9 \rho_{c}^{-5 / 3} \int_{0}^{M}\left(\gamma-\frac{4}{3}\right) P \frac{d m}{\varphi(m / M)}-1.84 \frac{G^{2} M^{7 / 3}}{c^{2}}=0 .
$$

These equations had been obtained by Bisnovatyi-Kogan (1966), using the expression of the energy with the prescribed distributions of the density (Emden polytrope $n=3$ ) and entropy (arbitrary) over the lagrangian coordinate $m / M$,

$$
\begin{gathered}
\epsilon=\int_{0}^{M} E(\rho, T) d m-\int_{0}^{M} \frac{G m d m}{r}-5.06 \frac{G^{2} M^{3}}{R^{2} c^{2}} \\
=\int_{0}^{M} E(\rho, T) d m-0.639 G M^{5 / 3} \rho_{c}^{1 / 3}-0.918 \frac{G M^{7 / 3}}{c^{2}} \rho_{c}^{2 / 3}, \\
d m=4 \pi \rho r^{2} d r
\end{gathered}
$$

by differentiation over the central density. Critical parameters of iron isentropic stellar cores for masses between 5 and $1000 M_{\odot}$ at the point of the loss of stability had been calculated by Bisnovatyi-Kogan and Kazhdan (1966). The dependence of the critical central density on mass is represented in Fig.6 (Bisnovatyi-Kogan, 2002) for a wide region of masses. At low masses, high densities the stability in the iron core is lost due to neutronization. For larger masses the temperature in the critical point is increasing, and stability is lost due to iron disintegration, decreasing the adiabatic power, and making it $\gamma \leq 1$. At masses 
around $1000 M_{\odot}$ with decreasing density and temperature, the adiabatic power becomes less than $4 / 3$ mainly due to pair creation, and at farther increasing of mass the effects of GR are the main reason of the loss of stability (Zeldovich and Novikov, 1965). At large masses the dependence $\rho_{c}(M)$ is described by the relation

$$
\rho_{c, \mathrm{cr}}(M)=2.4 \times 10^{17} \frac{1}{\mu^{3}}\left(\frac{M_{\odot}}{M}\right)^{7 / 2} \mathrm{~g} \mathrm{~cm}^{-3},
$$

where $\mu$ is the molecular weight.

Models of hot isentropic neutron stars had been calculated by Bisnovatyi-Kogan (1968), where equilibrium between iron, protons and neutrons was calculated, and the ratio of protons and neutrons was taken in the approximation of zero chemical potential of neutrino. The stability was checked using a variational principle in full GR (Chandrasekhar, 1964) with a linear trial function. The results of calculations, showing the stability region of hot "neutron" stars are given in Fig.7. Such stars may be called "neutron" only by convention, because they consist mainly from nucleons with almost equal presence of neutrons and protons. The maximum of the mass is about $70 M_{\odot}$, but from comparison of the total energies of hot neutron stars with presupernova cores we may conclude, that only collapsing cores with masses less that $15 M_{\odot}$ have a chance to stop at the state of a hot neutron star, and larger masses collapse directly to black holes.

The energetic method was generalized for rotating stars in the paper of Bisnovatyi-Kogan, Zeldovich and Novikov (1967), and in a more precise formulation by Bisnovatyi-Kogan and Ruzmaikin (1973). The density distribution remains the same, and the rotational energy term, written as a function of the total angular momentum was added. Rotational energy of the whole star depends on the central density as $\sim R^{-2} \sim \rho^{2 / 3}$, like the first GR correction term in (13). Therefore next order GR corrections had been taken into account $\sim R^{-3}$, which include also GR corrections to the internal and rotational energies. Calculations of the second GR corrections to the energy of nonrotating stars have been first done by Vartanyan (1972). Energetic method with two GR corrections for rotating stars was applied for investigation of stability of supermasive stars by Bisnovatyi-Kogan and Ruzmaikin (1973). It was obtained, that for large angular momenta rotation stabilizes the star against a collapse and prevent formation of a black hole. It is in correspondence with a fact, that Kerr solution for a rotating black hole exist only for sufficiently small angular momenta, and there are no black holes at larger values. Only radiation with $\gamma=4 / 3$ was taken into account in the equation of state, what lead to absence of equilibrium configurations at low angular 


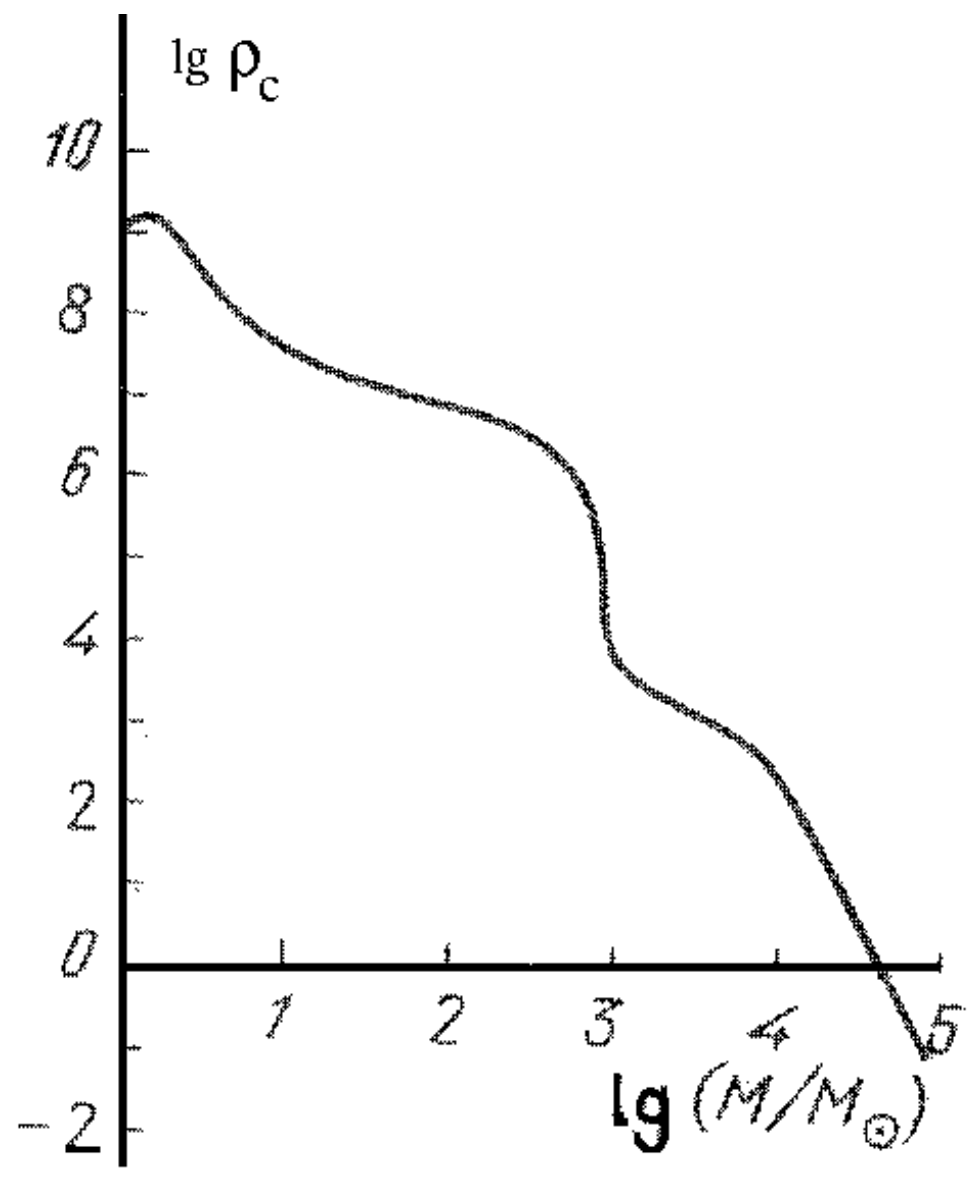

Figure 6. Central density as a function of stellar core mass for the critical states, from Bisnovatyi-Kogan (2002).

momenta $j$. The curves for equilibrium configurations at different $j$ are represented in Fig. 8 on the plane: equilibrium entropy $s_{e q}-x=$ $R_{g} / R$. The results are expressed in non-dimensional variables and are valid for an arbitrary mass star with the adiabatic equation of state $P=K \rho^{4 / 3}$. The energetic method gives asymptotically exact results for slowly rotating supermassive stars, but even at large $j$ the results remain correct qualitatively. ${ }^{1}$

\section{Search of quark stars}

At very high densities the equilibrium lowest energy state corresponds to quarks. However because of big indefiniteness of the theory the con- 


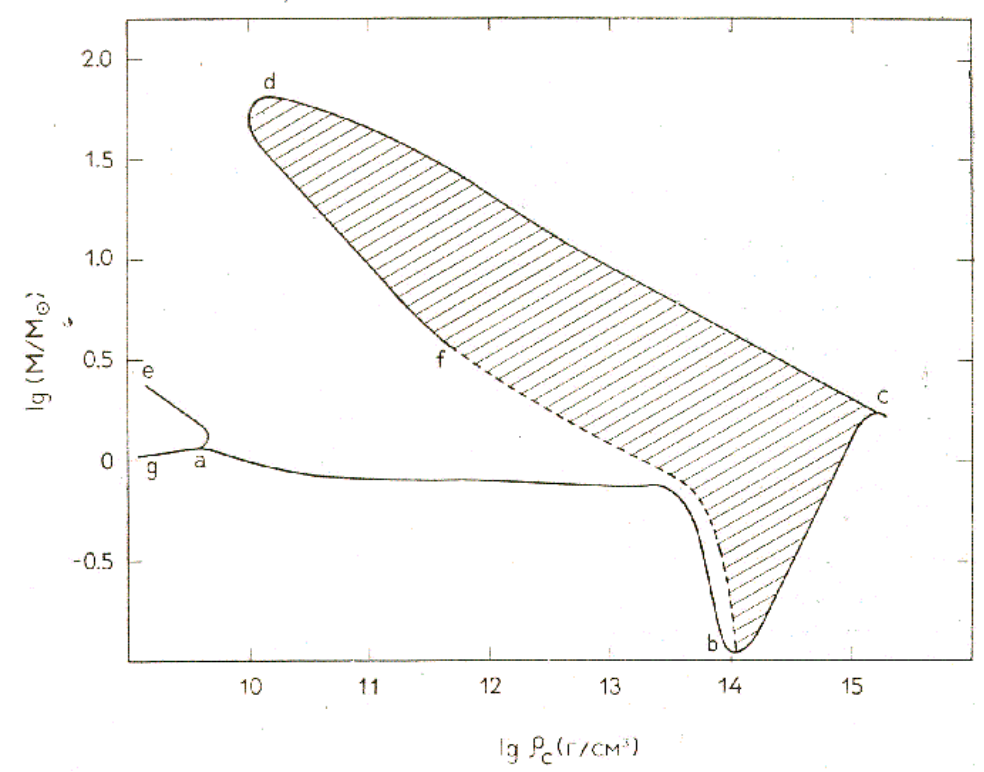

Figure \%. Equilibrium stable configurations on the mass $M$, central rest-mass density $\rho_{c}$ diagram for superdense isentropic stars (hatched). The stars become stable at sufficiently large densities because of nucleon pressure, and become unstable again at larger densities due to GR effects, from Bisnovatyi-Kogan (1968).

dition at which the quark state becomes preferable lays in a big density interval. Moreover, the process of transition between nucleon and quark state is also not definite, and even at very large densities the time of this transition may be enormously long. First the idea of a possibility of quark stars was suggested by Ivanenko and Kurdgelaidze (1965). Depending on the theory parameters there is a possibility of 3 types of quark stars: strange stars (SS), which consists of strange matter which may be stable at nuclear density with a zero pressure; hybrid stars with hadronic envelope, which has a core of pure quarks (QC), or has a mixed core of hadrons and quarks (MC). There are different approaches to describe a quark matter, see Aguirre and De Paoli (2002), Andersen and Strickland (2002), Burgio et al. (2002), Sedrakian and Blaschke (2002), Kohri et al. (2003), Lugones and Horvath (2003), Alford (2003), Mishustin et al. (2003). The results of calculation of hadronic $(\mathrm{H})$ and quark stellar models (SS, QC and MC) in Hard-Dense-Loop approach are represented in Fig.9 from Thoma et al. (2003), where one of a model parameters is changing. The free quarks exist in the state of deconfined quarks, and the density when deconfined quarks become energetically preferable is also rather indefinite (Berezhiani et al., 2003). 


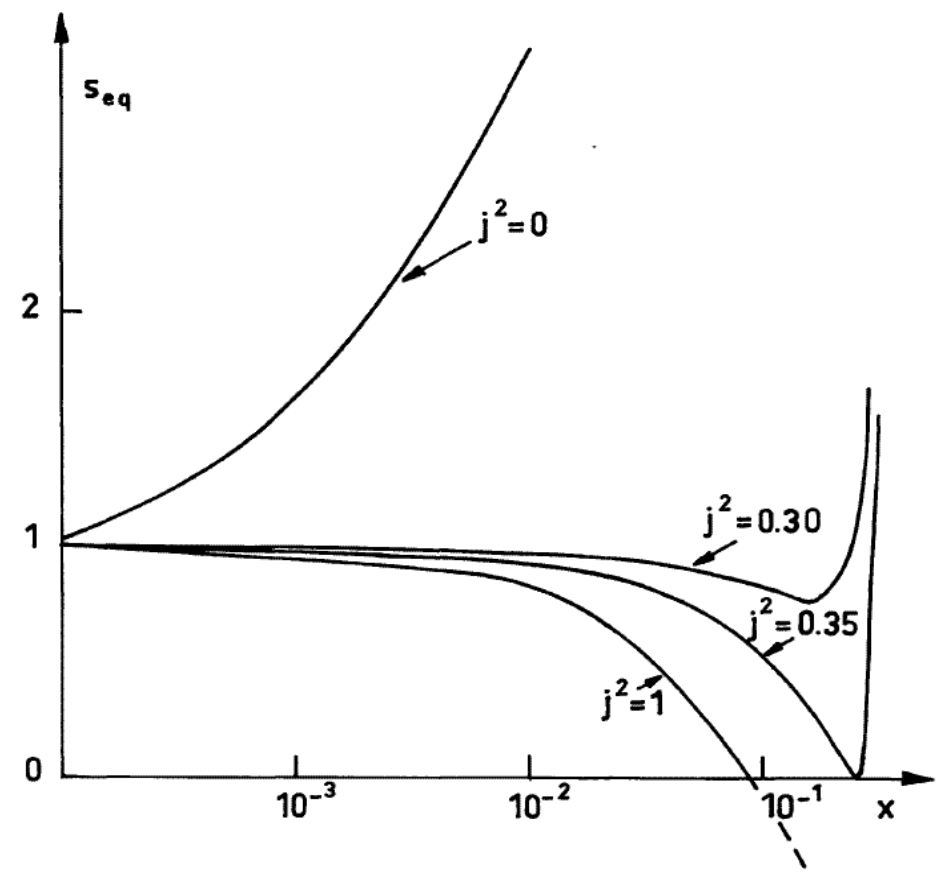

Figure 8. The curves $s_{e q}$ which characterizes the equilibrium entropy versus of $x=\frac{R_{g}}{R}$ for different values of the angular momentum $j$. The broken part corresponds to the nonphysical states $s_{e q}<0$. The parts of curves to the left of minima correspond to the stable states, from Bisnovatyi-Kogan and Ruzmaikin (1973).

Larger scattering of the properties of quark stars is obtained by Andersen and Strickland (2002), who used the same Hard-Dense-Loop approach, but with wider variation of parameters. Their models of quark stars are represented in Fig.10.

In presence of such problems with theory, only observations could give an answer about the possibility os existence of quark stars. Even in presence of theoretical indefiniteness, it is impossible to have a neutron (pure hadronic) star with a radius smaller than about 10 kilometers. Observational discovery of a compact star with considerably smaller radius would be an important evidence that this star is not pure hadronic. Such claim was made by Drake et al. (2002) basing on deep Chandra observations of the isolated compact star RX J1856.5-3754. They wrote: "We argue that the derived interstellar medium neutral hydrogen column density of $8 \times 10^{19} \leq N_{H} \leq 1.1 \times 10^{20} \mathrm{~cm}^{-2}$ favours the larger distance from two recent HST parallax analyses, placing RX J1856.5-3754 at $\sim 140 \mathrm{pc}$ instead of $\sim 60$ pc. $\ldots$ The combined observational evidence - a lack 


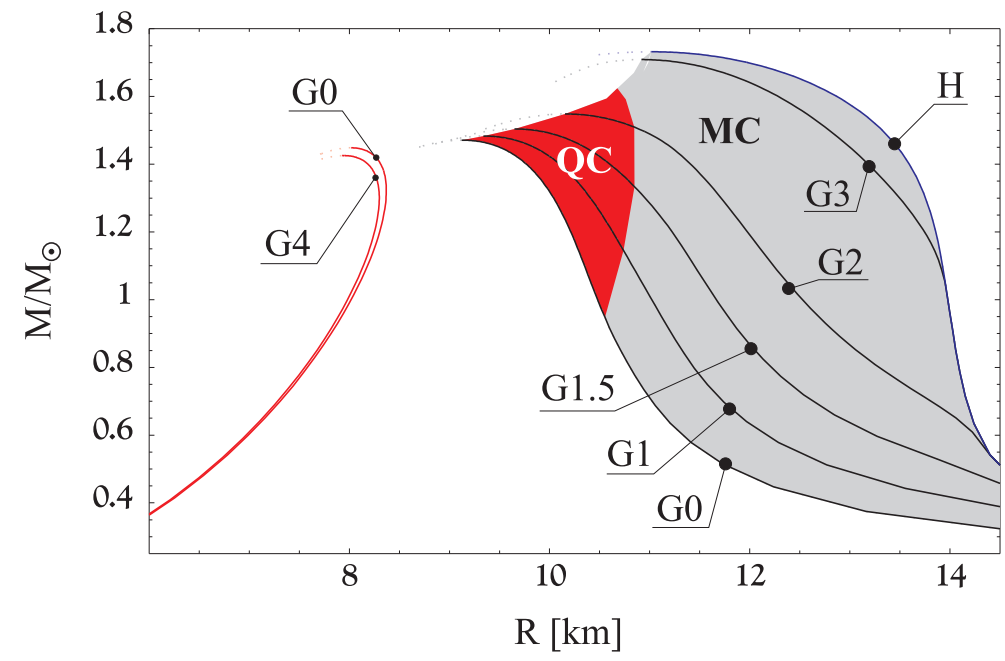

Figure 9. Mass-radius relation for pure strange quark matter stars (left) and hybrid stars (right). G0 - G4 models of hybrid stars corresponding to different parameters of the model. H: pure hadron star, QC: star has a quark core, MC: star has a mixed core, from Thoma et al. (2003).

of spectral and temporal features, and an implied radius $R_{\infty}=3.8$ $8.2 \mathrm{~km}$ that is too small for current neutron star models - points to a more compact object, such as allowed for quark matter equations of state." Subsequent analysis of observational data did not confirm this conclusion. Thoma et al. (2003) came to contrary result of an unusually large value of the radius even for a neutron star: "Combining such a X-ray spectrum with the optical spectrum, one finds a black-body radius $R_{\infty}>17 \mathrm{~km}$, indicating a stiff equation of state, which would exclude a strange quark matter star or even hybrid star. This is a rather conservative lower limit for the radius since a black-body emitter is the most efficient radiator." Similar conclusion was done by Turolla et al. (2003): "While a quark star may be a conceivable option, present observations do not necessarily demand this solution and more conventional scenarios involving a neutron star are certainly possible. Neutron star models based on a two-temperature surface distribution can account for both the X-ray and optical emission, giving at the same time acceptable values for the stellar radius." Meanwhile a new candidate for a quark (strange) star in the X-ray source 4U 1728-34 was suggested by Bombaci (2003), and farther discussion about this object is expected.

So far no reliable candidate for a quark (strange) star was found by observations. The theoretical predictions remain uncertain, and could explain either existence or nonexistence of quark stars. The solution 


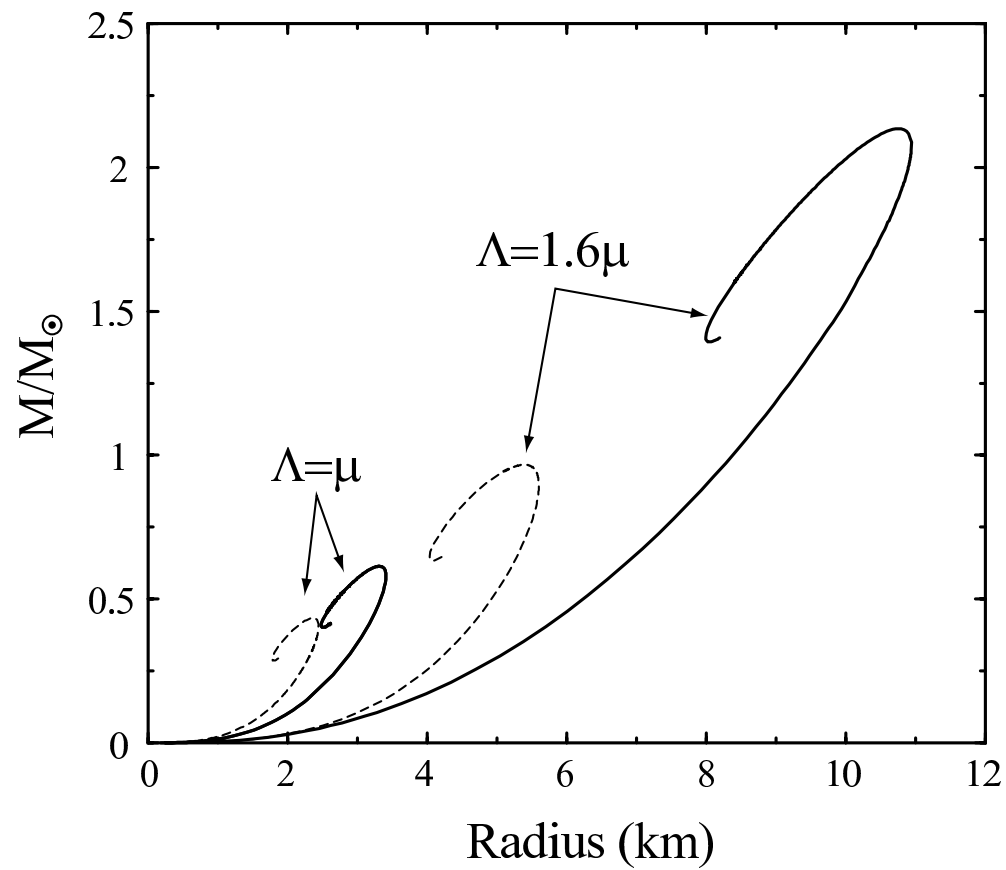

Figure 10. Mass-radius relation for a quark star with $\Lambda / \mu=1.6$ and $\Lambda / \mu=1$. The weak-coupling results for the same choice of renormalization scales are shown as dashed lines, from Andersen and Strickland (2002).

of intriguing problem of cosmological gamma ray burst (GRB) origin was proposed by Berezhiani et al (2003) basing on energy production during transformation of the neutron star into a stable quark (strange) star. This model could explain the connection of GRB with supernovae explosions with a formation of a neutron star, and subsequent huge energy release during transition to a state of a quark star producing GRB. An attractive feature of this model is a possibility of explanation of any time delay between SN and GRB explosions, which depends on the transition time from hadron to quark state, and is very sensitive to parameters. In this model the time delay may be arbitrary long, so majority of SN explosions in accordance with observations do not produce GRB, because this time exceeds the cosmological Hubble time.

\section{Summary}

1. Formation of quark (strange) stars does not follow unambiguously from the theory, which may be compatible either with existence or nonexistence of these objects. 
2. There are no firm observational contradictions to the conventional model of the hadronic neutron star.

3. Speculations about connection of the cosmological GRB with transition from the hadronic to quark star seems to be interesting, because they explain connection between GRB and supernovae explosion, with arbitrary time delay between these events, including very large, exceeding the Hubble time.

\section{Acknowledgments}

The author wish to thank D. Sedrakyan, D. Blaschke, G. Hadjan, Yu. Vartanyan, E. Chubaryan and A. Sadoyan for support and hospitality during the conference; and I.D. Novikov for useful discussion.

\section{Notes}

1. Because an approximate trial function does not give an absolute minimum of the energy functional, the loss of stability happens, strictly speaking, "before" the point obtained by the energetic method (EM), when going along the sequence of decreasing entropy or angular momentum. It means, that the model is definitely unstable in the EM critical point.

\section{References}

Aguirre, R. M., De Paoli, A. L. (2002). Neutron star structure in a quark model with excluded volume correction. nucl-th/0211038.

Alford, M. J. (2003). Dense quark matter in compact stars. nucl-th/030509\%.

Ambartsumyan, V. A., Saakyan, G.S. (1960). On degenerate superdense gas of elementary particles. Astron. Zh., 37: 193-209.

Ambartsumyan, V. A., Saakyan, G.S. (1961). Internal structure of hyperonic configurations of stellar masses. Astron. Zh., 38: 1016-1024.

Andersen, J. O., Strickland, M. (2002) The Equation of State for Dense QCD and Quark Stars. Phys.Rev., D66: 105001-105005.

Baade, W., Zwicky, F. (1934). Supernovae and cosmic rays. Phys. Rev., 45: 138-139.

Berezhiani, Z., Bombaci, I., Drago, A., Frontera, F., Lavagno, A. (2003). Gamma Ray Bursts from delayed collapse of neutron stars to quark matter stars. Astrophys.J., 586: $1250-1253$.

Bisnovatyi-Kogan, G. S. (1966). Critical mass of hot isothermal white dwarf with the inclusion of general relativity effects. Astron. Zh., 43: 89-95.

Bisnovatyi-Kogan, G. S. (1968). The mass limit of hot superdense stable configurations. Astrofizika, 4: 221-238.

Bisnovatyi-Kogan, G. S. (2002). Stellar Physics. Vol.2. Stellar evolution and stability. Berlin Heidelberg: Springer.

Bisnovatyi-Kogan,G.S., Blinnikov,S.I. (1974). Static criteria for stability of arbitrary rotating stars. Astron. Ap., 31: 391-404.

Bisnovatyi-Kogan, G. S., Blinnikov, S. I., Shnol', E. E. (1975). The stability of a star in the presence of a phase transition. Astron. Zh., 52: 920-929.

Bisnovatyi-Kogan, G. S., Kajdan, Ya. M. (1966). Critical parameters of stars. Astron. Zh., 43: 761-771. 
Bisnovatyi-Kogan,G.S., Ruzmaikin,A.A. (1973). The stability of rotating supermassive stars. Astron.Ap., 27: 209-221.

Bisnovatyi-Kogan, G. S., Zel'Dovich, Ya. B., Novikov, I. D. (1967). Evolution of supermassive stars stabilized by large-scale motions. Astron. Zh., 44: 525-536.

Bombaci, I. (2003). A possible signature for quark deconfinement in the compact star in $4 \mathrm{U}$ 1728-34. astro-ph/0307522.

Burgio, G. F., Baldo, M., Schulze, H.-J., Sahu, P. K. (2002). The hadron-quark phase transition in dense matter and neutron stars. Phys.Rev., C66: 025802-025815.

Cameron, A. G. (1959). Neutron star models. Astrophys. J., 130: 884-894.

Chandrasekhar, S. (1931). The maximum mass of ideal white dwarf. Astrophysical J., 74: 81-82.

Chandrasekhar, S. (1964). The dynamical instability of gaseous masses approaching the Schwarzschild limit in general relativity. Astrophys. J., 140: 417-433.

Comella, J. M., Craft, H. D. Jr., Lovelace, R. V. E., Sutton, J.M. and Tyler, G.I. (1969). Crab nebula pulsar NP 0532. Nature, 221: 453-454.

Dmitriyev, N. A. and Kholin, S. A. (1963). Features of static solutions of the gravity equations. Voprosy Kosmogonii, 9: 254-262.

Drake, J. J., Marshall, H. L.. Dreizler, S., Freeman, P. E., Fruscione, A., Juda, M., Kashyap, V., Nicastro, F., Pease, D. O., Wargelin, B. J., Werner, K. (2002) Is RX J1856.5-3754 a Quark Star? Astrophys. J., 572: 996-1001.

Emden, R. (1907). Gaskugeln. Leipzig.

Gamow, G. (1938). Zusammenfassender Bericht. Kernumwandlungen als Energiequelle der Sterne. Zeitschrift für Astrophysik, 16: 113-160.

Harrison, B. K., Thorne, K. S., Wakano, M., Wheeler,J.A. (1965). Gravitational Theory and Gravitational Collapse Chicago, University of Chicago Press.

Hund, F. (1936). Ergebn. Exakt. Naturwiss., 15: 189-212.

Ivanenko, D.D., Kurdgelaidze, D.F. (1965). Astrofizika, 1: 251-253.

Kohri, K., Iida, K., Sato, K. (2003). Upper limit on the mass of RX J1856.5-3754 as a possible quark star. Prog. Theor.Phys., 109: 765-780.

Landau, L. D. (1932). On the theory of stars. Phys. Zeit. Sov., 1: 285-288.

Landau, L. D. (1938). Nature, 141: 333-334.

Lovelace, R. V. E., Sutton, J.M., Craft, H. D. Jr. (1968). Pulsar NP 0532 near Crab nebula. IAU Circular No. 2113.

Lugones, G., J.E. Horvath, J.E. (2003). Quark-diquark equation of state and compact star structure. Int. J. Mod. Phys., D12: 495-508.

Mishustin, I.N., Hanauske, M., Bhattacharyya, A., Satarov, L.M., Stoecker, H., Greiner, W. (2003). Ca tastrophic re arrangement of a compact star due to the quark core formation. Phys.Lett., B552: 1-8.

Misner, C. W.; Zapolsky, H. S. (1964). High-Density Behavior and Dynamical Stability of Neutron Star Models. Phys. Rev. Let., 12: 635-637.

Oppenheimer, J. R.; Volkoff, G. M. (1939). On Massive Neutron Cores. Phys. Rev., 55: $374-381$.

Rosenfeld, L. (1974). Astrophys. and gravitation. in Proc. 16 Solvay Conf. on Phys, Univ. de Bruxells, p. 174.

Rudkjöbing, M. (1952). Publ. Köbenhavns Obs., No.160.

Saakyan, G. S., Vartanyan, Yu. L. (1964). Basic Parameters of Baryon Configurations. Astron. Zh., 41: 193-201.

Sedrakian, D. M., Blaschke, D. (2002). Magnetic field of a neutron star with color superconducting quark matter core. Astrofiz., 45: 203-212.

Skryme, T. H. R. (1959). Nucl. Phys., 9: 615-625. 
Thoma, M.H., Trümper, J., Burwitz, V. (2003). Strange Quark Matter in Neutron Stars? - New Results from Chandra and XMM. astro-ph/0305249.

Turolla, R., Zane, S., Drake, J.J. (2003). Bare Quark Stars or Naked Neutron Stars: The Case of RX J1856.5-3754. astro-ph/0308326.

Vartanyan, Yu. L. (1972). Doctor. Diss., Yerevan.

Wheeler, J.A. (1958). Paper read at 11 Solvay conference, Brussels. Published with Harrison, B.K. and Wakano, M. in La structure et l'evolution de l'universe. (Brussels: R. Stoops.)

Zel'dovich,Ya.B. (1961). Equation of state at a superhigh density and relativistic restrictions. Journ. Exp. Theor. Fiz. 41: 1609-1615.

Zel'dovich, Ya. B. (1963). Hydrodynamical stability of star. Voprosy Kosmogonii, 9: $157-170$.

Zel'dovich, Ya. B., Novikov, I. D. (1965). Relativistic astrophysics. II. Uspekhi. Fiz. Nauk, 86: 447-536. 


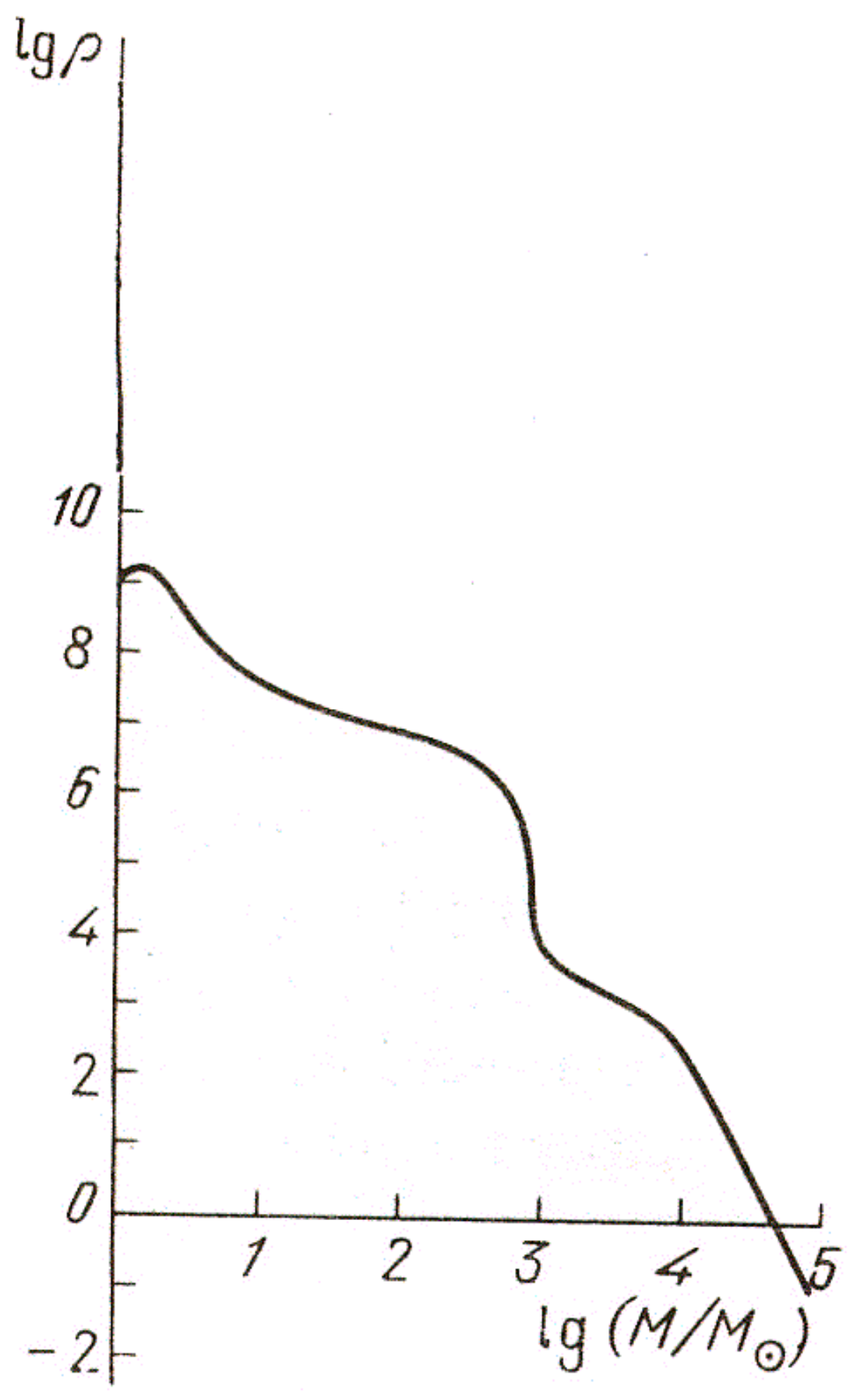

